\title{
Associations of the hypertension-related single nucleotide polymorphism rs11191548 with high-density lipoprotein cholesterol and leptin in Chinese children
}

\author{
Lijun $\mathrm{Wu}^{1+} \mathbb{D}$, Liwang $\mathrm{Gao}^{1+}$, Xiaoyuan Zhao ${ }^{1}$, Meixian Zhang ${ }^{1}$, Jianxin $\mathrm{Wu}^{2}$ and Jie $\mathrm{Mi}^{1 *}$
}

\begin{abstract}
Background: The genome-wide association study has founded hypertension-related single nucleotide polymorphism (SNP) rs11191548 near CYP17A1 encoding a key enzyme involved in steroid metabolism, but the molecular mechanisms are not understood and the associations of the SNP with hypertension-related traits are not fully described, especially in children. The aim of the present study is to investigate the associations between the SNP and two hypertension-related traits, lipids and leptin.
\end{abstract}

Methods: We genotyped the SNP in Beijing Child and Adolescent Metabolic Syndrome (BCAMS) study. A total of 3503 children participated in the study.

Results: The SNP rs11191548 was significantly associated with high-density lipoprotein cholesterol (HDL) $(P=0.014$ and 0. 028, respectively) and leptin ( $P=0.011$ and 0.026 , respectively) under an additive model after adjustment for age, gender, and systolic blood pressure (SBP) or diastolic blood pressure (DBP). There was a statistically significant association of rs11191548 with high leptin after adjustment for age, gender, and SBP or DBP. The $P$-values remain significant after correction for multiple testing.

Conclusions: We demonstrate for the first time that the SNP rs11191548 near CYP17A1 is associated with HDL and leptin in Chinese children. These novel findings provide important evidence that HDL and leptin maybe possibly mediate the process of CYP17A1 involved in hypertension.

Keywords: Leptin, High-density lipoprotein cholesterol, CYP17A1, Hypertension-related single nucleotide polymorphism

\section{Background}

In recent years, the prevalence of hypertension has been increasing in most parts of the world, and hypertension is a major threat to public health [1]. Childhood hypertension is a predictor of adult hypertension and cardiovascular disease $[2,3]$.

Previously, multiple single nucleotide polymorphisms (SNPs) related to hypertension have been identified by genome-wide association studies [4-6]. Among those identified SNPs, the SNP rs11191548, located near the

\footnotetext{
*Correspondence: Jiemi@vip.163.com

${ }^{\dagger}$ Equal contributors

'Department of Epidemiology, Capital Institute of Pediatrics, No. 2 Yabao

Road, Chaoyang District, Beijing 100020, China

Full list of author information is available at the end of the article
}

3 ' noncoding region of the gene CYP17A1 encoding the cytochrome P450 enzyme CYP17A1as a key enzyme involved in steroid metabolism, showed a significant association with hypertension in European adults, Japanese and Chinese adults, and Chinese children [6-9]. However, the molecular mechanisms are not understood and the associations of the SNP with other hypertension -related traits are not fully described.

Dyslipidemia are recognized as a strong predictor of cardiovascular disease and several studies have suggested a positive correlation between dyslipidemia and hypertension $[10,11]$. Leptin, an adipocytokine produced by adipose tissue, is associated with dyslipidemia [12], and recent study has demonstrated that leptin contributes to 
Table 1 Associations of rs11191548 with HDL and leptin

\begin{tabular}{|c|c|c|c|c|c|c|c|c|c|c|c|c|c|c|c|}
\hline \multirow{2}{*}{$\begin{array}{l}\text { Additive } \\
\text { model }\end{array}$} & \multirow[b]{2}{*}{$\mathrm{N}$} & \multicolumn{7}{|l|}{$\mathrm{HDL}$} & \multicolumn{7}{|l|}{ Leptin } \\
\hline & & Mean \pm SD & $P$-value & Power & $P$-value & Power & $P$-value & $\overline{\text { Power }}$ & Mean \pm SD & $P$-value ${ }^{a}$ & Power & $P$-value & Power & $P$-value ${ }^{c}$ & $\overline{\text { Power }}$ \\
\hline$\overline{C C}$ & 260 & $1.36 \pm 0.32$ & & & & & & & $10.71 \pm 12.72$ & & & & & & \\
\hline CT & 1380 & $1.40 \pm 0.32$ & & & & & & & $10.42 \pm 11.63$ & & & & & & \\
\hline$\pi$ & 1791 & $1.41 \pm 0.32$ & 0.043 & 0.552 & $0.014^{d}$ & 0.691 & $0.028^{d}$ & 0.600 & $9.70 \pm 11.14$ & 0.051 & 0.558 & 0.011 & 0.745 & 0.026 & 0.646 \\
\hline
\end{tabular}

HDL high-density lipoprotein cholesterol, SD standard deviation

${ }^{a}$ Adjusted for age and gender, ${ }^{b}$ Adjusted for age, gender and SBP, ${ }^{c}$ Adjusted for age, gender and DBP, ${ }^{d} P$-value remains significant after FDR test is applied

hypertension through upregulation of central reninangiotensin system and proinflammatory cytokines [13].

There has been no evidence that the SNP rs11191548 near CYP17A1 is associated with lipids and leptin. We investigated the associations between the SNP rs11191548 with lipids and leptin in the cohort. We genotyped the SNP in Chinese children who were participated in the populationbased Beijing Child and Adolescent Metabolic Syndrome (BCAMS) study. The present study attempts to provide an analysis of epidemiological and genetic data towards the possible mechanism of the role of CYP17A1 in hypertension.

\section{Methods}

\section{Study population}

Subjects were recruited from a cross-sectional populationbased survey termed the BCAMS study in 2004. The survey included a questionnaire, anthropometric measurement, and medical examination in a representative sample $(n=$ $19,593,50 \%$ boys) of children in Beijing aged 6-18 years. Anthropometric measurements included weight, height, waist circumference, and fat mass percentage. Within this large group of children, 1045 children with elevating blood pressure (including pre-hypertension and hypertension) and 2458 children with normal blood pressure were randomly recruited and diagnosed by using blood pressure reference cutoffs for Chinese children and adolescents [14]. Venipuncture blood samples were collected for genotyping. The BCAMS study was approved by the ethics committees of Capital Institute of Pediatrics. We obtained written informed consent from parents or guardians.

\section{Measurement of biochemical analyses and genotyping}

Total cholesterol (TC), low-density lipoprotein cholesterol (LDL), high-density lipoprotein cholesterol (HDL), triglycerides (TG), were analyzed by an automatic biochemical analyzer (Hitachi 7060) using a kit assay (SEKISUI medical technology Ltd., Tokyo, Japan). The levels of adipocytokines were measured by ELISA techniques [15]. Genomic DNA was isolated from peripheral white blood cells using the salt fractionation method. Genotyping of rs11191548 was conducted using the TaqMan Allelic Discrimination Assay with the GeneAmp 7900 Sequence Detection System (Applied Biosystems, Foster City, CA, USA), with the TaqMan probes (C_31979323_10). The genotyping call rate for the SNP was $97.9 \%$. We sent 30 samples to direct sequencing and observed $100 \%$ concordance between two genotyping methods. We also repeated 70 samples randomly for the SNP to validate the accuracy of genotyping and observed $100 \%$ concordance between the results of the two tests.

\section{Statistical analysis}

Categorical variables were presented as percentages and continuous variables were presented as mean \pm standard deviation (SD). Hardy-Weinberg equilibrium was assessed using the chi-square test. Adjusted odds ratios (ORs) for high leptin were performed by logistic regression with genotypes, age, gender, and systolic blood pressure (SBP) or diastolic blood pressure (DBP) as the independent variables. The data were analysed using SPSS statistical software. $P<0.05$ was used to indicate statistically significant differences. False discovery rate (FDR) approach was used to correct for multiple testing. In brief, the stringent $p$ value was considered statistically significant only if it was less than 0.05. Power calculation was performed using Quanto software according to the assumed effect size and allele frequency (http://hydra.usc.edu/gxe/).

\section{Results}

The basic characteristics of the study participants are summarized in Table S1 (Additional file 1). We genotyped the SNP rs11191548 near CYP17A1 in the cohort, and the genotype of the SNP was tested to be in Hardy-Weinberg equilibrium $(P=0.795)$. The minor allele frequency (MAF) of the SNP was 0.277 in the cohort. The associations of the SNP rs11191548 with HDL and leptin are shown in Table 1. As the SNP was associated with blood

Table 2 Association of rs11191548 with high leptin under the additive model

\begin{tabular}{|c|c|c|c|c|c|c|c|c|c|c|c|c|}
\hline \multirow[t]{2}{*}{ Factors } & \multicolumn{4}{|c|}{ Adjusted for age and gender } & \multicolumn{4}{|c|}{ Adjusted for age, gender and SBP } & \multicolumn{4}{|c|}{ Adjusted for age, gender and DBP } \\
\hline & $\mathrm{OR}$ & $95 \% \mathrm{Cl}$ & $P$-value & Power & OR & $95 \% \mathrm{Cl}$ & $P$-value & Power & OR & $95 \% \mathrm{Cl}$ & P-value & Power \\
\hline High leptin & 0.874 & $0.782-0.978$ & $0.019^{\mathrm{a}}$ & 0.700 & 0.841 & $0.749-0.945$ & $0.004^{\mathrm{a}}$ & 0.889 & 0.855 & $0.762-0.960$ & $0.008^{\mathrm{a}}$ & 0.822 \\
\hline
\end{tabular}

$S B P$ systolic blood pressure, $D B P$ diastolic blood pressure, $O R$ odds ratio, $C l$ confidence interval

a $P$-value remains significant after FDR test is applied 
pressure, we also adjusted SBP or DBP besides age and gender. The SNP rs11191548 was significantly associated with HDL under an additive model $(P=0.014$ and 0.028 , respectively) after adjustment for age, gender, and SBP or DBP. The SNP was also significantly associated with leptin under an additive model $(P=0.011$ and 0.026 , respectively) after adjustment for age, gender, and SBP or DBP.

Table 2 shows the association of rs11191548 with high leptin defined as leptin $\geq$ the 75 percentile of the participant with same age and gender. The SNP rs11191548 was significantly associated with high leptin under an additive model after adjustment for age, gender, and SBP or DBP. The P-values remain significant after correction for multiple testing.

\section{Discussion}

CYP17A1 encodes the cytochrome P450 enzyme CYP17A1 and has a key role in the biosynthesis of steroid hormones [16]. CYP17A1 deficiency caused by a mutation in the gene usually results severe hypertension and hypokalemia in males [17]. The SNP rs11191548 near CYP17A1 showed a significant association with hypertension [6-9], but the molecular mechanisms are not understood.

In this study, we examined the SNP rs11191548 near CYP17A1 with lipids and leptin in Chinese children. Our results indicated that the SNP rs11191548 was significantly associated with HDL and leptin after adjustment for age, gender, and SBP or DBP, and there was statistically significant association of the SNP rs11191548 with high leptin under an additive model adjusted for age, gender, and SBP or DBP, after correction for multiple testing. No significant associations of the SNP with TC, TG and LDL were found in the population (data not shown). Studies with greater sample size are needed to confirm these associations.

This study may not provide direct evidence that the expression of CYP17A1 influences hypertension because the lack of gene expression data, but our study demonstrated the associations of the SNP rs11191548 near CYP17A1 with HDL and leptin that contribute to hypertension.

\section{Conclusion}

We demonstrate for the first time that the SNP rs11191548 near CYP17A1 is associated with HDL and leptin in Chinese children. These novel findings provide important evidence that HDL and leptin maybe possibly mediate the process of CYP17A1 involved in hypertension.

\section{Additional file}

Additional file 1: TAble S1. Basic characteristics of study participants (DOC $42 \mathrm{~kb}$ )

\section{Abbreviations}

DBP: Diastolic blood pressure; FDR: False discovery rate; HDL: High-density lipoprotein cholesterol; LDL: Low-density lipoprotein cholesterol; ORs: Odds ratios; SBP: Systolic blood pressure; SD: Standard deviation; SNPs: Single nucleotide polymorphisms; TC: Total cholesterol; TG: Triglycerides

\section{Acknowledgements}

The authors thank the children for their participation in this study. We also thank Xuejun Ma, Chinese Center for Disease Control and Prevention, Beijing, China, for providing the genotyping facilities for our work.

\section{Funding}

This study was supported by the National Basic Research Program of China (973 Program, grant no. 2013CB530605), the National Natural Science Foundation of China (grant no. 81473062) and the Beijing Health System High-Level Technical Talents Training Fund (grant no. 2015-3-083).

\section{Availability of data and materials}

All data generated or analysed during this study are included in this published article.

\section{Authors' contributions}

LW designed the study, collected the data and wrote the manuscript. LG performed the statistical analysis. XZ and MZ collected the DNA samples. All the authors reviewed and approved the final manuscript. JM directed the project.

\section{Ethics approval and consent to participate}

The BCAMS study was approved by the ethics committees of Capital Institute of Pediatrics. We obtained written informed consent from parents or guardians.

\section{Consent for publication}

Not applicable.

\section{Competing interests}

The authors declare that they have no competing interests.

\section{Publisher's Note}

Springer Nature remains neutral with regard to jurisdictional claims in published maps and institutional affiliations.

\section{Author details}

'Department of Epidemiology, Capital Institute of Pediatrics, No. 2 Yabao Road, Chaoyang District, Beijing 100020, China. ${ }^{2}$ Department of Biochemistry, Capital Institute of Pediatrics, No. 2 Yabao Road, Chaoyang District, Beijing 100020, China.

Received: 8 May 2017 Accepted: 9 January 2018

Published online: 16 January 2018

\section{References}

1. Mittal BV, Singh AK. Hypertension in the developing world: challenges and opportunities. Am J Kidney Dis. 2010;55:590-8.

2. Chen $X$, Wang Y. Tracking of blood pressure from childhood to adulthood: a systematic review and meta-regression analysis. Circulation. 2008;117: 3171-80.

3. Santi M, Simonetti BG, Leoni-Foglia CF, Bianchetti MG, Simonetti GD. Arterial hypertension in children. Curr Opin Cardiol. 2015;30:403-10.

4. Padmanabhan S, Caulfield M, Dominiczak AF. Genetic and molecular aspects of hypertension. Circ Res. 2015;1 16:937-59.

5. Lu X, Wang L, Lin X, Huang J, Charles Gu C, et al. Genome-wide association study in Chinese identifies novel loci for blood pressure and hypertension. Hum Mol Genet. 2015;24:865-74.

6. Newton-Cheh C, Johnson T, Gateva V, Tobin MD, Bochud M, et al. Genomewide association study identifies eight loci associated with blood pressure. Nat Genet. 2009:41:666-76.

7. Takeuchi F, Isono M, Katsuya T, Yamamoto K, Yokota M, et al. Blood pressure and hypertension are associated with 7 loci in the Japanese population. Circulation. 2010;121:2302-9. 
8. Liu C, Li H, Qi Q, Lu L, Gan W, et al. Common variants in or near FGF5, CYP17A1 and MTHFR genes are associated with blood pressure and hypertension in Chinese Hans. J Hypertens. 2011;29:70-5.

9. Wu L, Xi B, Zhang M, Shen Y, Zhao X, et al. A sex-specific effect of the CYP17A1 SNP rs11191548 on blood pressure in Chinese children. J Hum Hypertens. 2012;26:731-6.

10. Otsuka T, Takada H, Nishiyama Y, Kodani E, Saiki Y, et al. Dyslipidemia and the risk of developing hypertension in a working-age male population. J Am Heart Assoc. 2016;5:e003053.

11. Borghi C, Rodriguez-Artalejo F, De Backer G, Dallongeville J, Medina J, et al. The association between blood pressure and lipid levels in Europe: European study on cardiovascular RiskPrevention and Management in Usual Daily Practice. J Hypertens. 2016;34:2155-63.

12. de Haro MC, Figueiredo VN, de Faria AP, Barbaro NR, Sabbatini AR, et al. High-circulating leptin levels are associated with increased blood pressure in uncontrolled resistanthypertension. J Hum Hypertens. 2013;27:225-30.

13. Xue B, Yu Y, Zhang Z, Guo F, Beltz TG, et al. Leptin mediates high-fat diet sensitization of Angiotensin II-elicited hypertension by Upregulating the BrainRenin-Angiotensin system and inflammation. Hypertension. 2016;67:970-6.

14. Xi B, Zong X, Kelishadi R, Hong YM, Khadilkar A, et al. Establishing international blood pressure references among nonoverweight children and adolescents aged 6 to 17 years. Circulation. 2016;133:398-408.

15. Araki S, Dobashi K, Kubo K, Asayama K, Shirahata A. High molecular weight, rather than total, adiponectin levels better reflect metabolic abnormalities associated with childhood obesity. J Clin Endocrinol Metab. 2006;91:5113-6.

16. Dhir V, Ivison HE, Krone N, Shackleton CH, Doherty AJ, et al. Differential inhibition of CYP17A1 and CYP21A2 activities by the P450 oxidoreductase mutant A287P. Mol Endocrinol. 2007;21:1958-68.

17. Yang J, Cui B, Sun S, Shi T, Zheng S, et al. Phenotype-genotype correlation in eight Chinese 17alpha-hydroxylase/17,20 lyase-deficiency patients with five novel mutations of CYP17A1 gene. J Clin Endocrinol Metab. 2006;91: 3619-25.

\section{Submit your next manuscript to BioMed Central and we will help you at every step:}

- We accept pre-submission inquiries

- Our selector tool helps you to find the most relevant journal

- We provide round the clock customer support

- Convenient online submission

- Thorough peer review

- Inclusion in PubMed and all major indexing services

- Maximum visibility for your research

Submit your manuscript at www.biomedcentral.com/submit

) Biomed Central 